\title{
RAPID VARIABILITY OF GAMMA-RAY BLAZARS: A MODEL FOR MARKARIAN 421
}

\author{
M. SALVATI ${ }^{1}$ M. SPADA ${ }^{2}$ AND F. PACINI ${ }^{1,2}$
}

Received —

${ }^{1}$ Osservatorio Astrofisico di Arcetri, L. E. Fermi 5, I-50125 Firenze, Italy

${ }^{2}$ Dipartimento di Astronomia e Scienza dello Spazio, Università di Firenze, L. E. Fermi 5, I-50125 Firenze, Italy 


\begin{abstract}
The extremely rapid burst of TeV photons from Mkn 421 (15 May 1996) can be reconciled with the standard properties of a relativistic $\gamma$-ray emitting jet (bulk Lorentz factor $\Gamma \sim 10$; size $\sim 10^{17} \mathrm{~cm}$ ) if one assumes that the electrons are accelerated in conical shocks with both opening and viewing angles $\approx 1 / \Gamma$. If the injection time and the cooling time are much less than the photon crossing time, an emission ring moves along the jet and leads to the appearance of a very rapid flare, in satisfactory agreement with the observations.
\end{abstract}

Subject headings: BL Lacertae objects : individual (Mkn 421) — galaxies : jets — gamma rays : observations — radiation mechanisms : non thermal 


\section{INTRODUCTION}

Several blazars, all of them associated with radio loud AGN, show an intense and variable $\gamma$-ray emission (Hartman et al. 1992).

Various authors (Maraschi, Ghisellini \& Celotti 1992; Dermer, Schlickeiser \& Mastichiadis 1992; Sikora, Begelman \& Rees 1994; Inoue \& Takahara 1996) have shown that the observed luminosity - up to $\sim 10^{48} \operatorname{ergs~s}^{-1}$ or more - and variability - typical time scales $\sim$ several hours - can be understood if the emission is due to inverse Compton scattering of relativistic electrons on softer photons inside a relativistic jet. These jets move with bulk Lorentz factor $\Gamma \sim 5-10$, at a distance from the nucleus of order $10^{16}-10^{17} \mathrm{~cm}$ : they appear to be the inner part of the well known radio jets, with similar $\Gamma$ but larger sizes $\left(10^{19}-10^{20} \mathrm{~cm}\right)$. Since the $\gamma$-ray emission occurs closer to the central objects, it provides direct information about the physical processes effective in the central region of AGN.

In this connection, the discovery of large (factor of order 20) and very rapid (time scale around 20 minutes) variations of the TeV emission from Mkn 421 on 15 May 1996 (Gaidos et al. 1996) represents an important clue to the physical conditions in the central region of AGN. Variability timescales of 20 minutes would entail a source size $\approx 10^{15} \mathrm{~cm}$ (with problems for photon-photon opacity) or $\Gamma \gtrsim 10^{2}$ (with problems for the total energy and the low probability of observing an event with a visibility cone $\sim 1 / \Gamma$ ).

Our purpose in this Letter is to present a model for the occurrence of rapid variations which uses the standard scenario but does not require extreme values for the Lorentz factor $\Gamma$ or the size. The variability is related to anisotropies in the comoving frame of the jet associated with the geometry of the shocks responsible for the particle acceleration. We shall show that strong spikes in the very high energy spectrum are a natural consequence of a nearly conical shock geometry with characteristic opening $\approx 1 / \Gamma$. 


\section{RELEVANT TIME SCALES}

We shall assume that the jet has a bulk Lorentz factor $\Gamma \gg 1$, scale length $z$ and radius $r$; the scale length is defined as the distance over which the bulk properties of the jet change substantially: let $\Phi$ be one such property, then $z=\left|\frac{\Phi}{d \Phi / d z}\right|$. The observer's line of sight makes an angle $\theta \approx 1 / \Gamma$ to the jet axis.

The time scales relevant to the variability are the following ones, as measured by a distant observer:

1. the geometrical time needed to the photons to cross the emission region either in length (longitudinal time $\Delta t_{z} \approx z / c \Gamma^{2}$ ) or in width (radial time $\Delta t_{r} \approx r / c \Gamma$ );

2. the radiative time $\Delta t_{e m}$ over which the emitting electrons lose a large fraction of their energy; if $\gamma$ is the typical electron Lorentz factor in the comoving frame, the dot indicates the time derivative, and the prime refers to quantities measured in the comoving frame, then $\Delta t_{e m} \approx(\gamma / \dot{\gamma})^{\prime} / \Gamma$

3. the injection time $\Delta t_{\text {in }}$ over which the electrons are fed to the emission region; as will be shown in the following, the radiative time is typically shorter than the geometrical time, and the electrons must be accelerated in situ; there are arguments (Blandford) \& Levinson 1995) which point to the electrons being not only accelerated, but also generated in situ.

From the point of view of the observer, a variability episode appears convolved with $\Delta t_{i n}$, $\Delta t_{e m}, \Delta t_{r}$, and is dominated by the longest of them. On the other hand, in a diverging jet the emission mechanisms become very rapidly ineffective over distances longer than $z$, so that $\Delta t_{z}$ is an upper bound to the observed variability $\Delta t_{o b s}$ 


$$
\Delta t_{o b s} \approx \min \left[\Delta t_{z}, \max \left(\Delta t_{i n}, \Delta t_{e m}, \Delta t_{r}\right)\right]
$$

There are various arguments which suggest that $r \approx z / \Gamma$; if this is indeed the case, and $\Delta t_{r} \approx \Delta t_{z}$ (as we shall assume in the following), $\Delta t_{o b s}$ is necessarily of the order of the longitudinal time $\Delta t_{z}$. In this paper we show how a plausible geometric arrangement might release this constraint, otherwise unavoidable.

The injection time scale $\Delta t_{\text {in }}$ is very likely the shortest of all, of the order of several minutes, depending on the mass one chooses for the central black hole. The remaining time scales $\Delta t_{e m}$ and $\Delta t_{z}$ are related in an interesting way to the jet optical depth to photon-photon absorption. This is due to the near equality of the cross sections involved, and to the radiation density in the jet being the target for both the emission and the absorption processes. The relation can be expressed in closed form under certain assumptions: only the jet contributes to the radiation field, the radiation spectrum is flat in $\nu F_{\nu}$, the scattering process is always in the Thomson regime. Then one has

$$
\ell=\frac{L \sigma_{T}}{m c^{3} z}, \quad \frac{\Delta t_{e m}}{\Delta t_{z}}=\frac{3 \pi}{4 \ell} \frac{\Gamma^{3}}{\gamma}, \quad \tau_{\gamma \gamma}=\frac{3 \ell}{16 \pi} \frac{\epsilon_{u}}{\Gamma^{4}}\left(\ln \frac{\epsilon_{\mathrm{u}}}{\epsilon_{\mathrm{d}}}\right)^{-1}
$$

The quantity $\ell$ is the compactness of the jet radiation field in terms of the observed luminosity $L, \tau_{\gamma \gamma}$ is the jet photon-photon optical depth, and $\epsilon_{u}$ and $\epsilon_{d}$ are the upper and lower energies of the photon spectrum, respectively, in units of $m c^{2}$. By multiplying the second and third of Eqs. 2 we finally get

$$
\frac{\Delta t_{e m}}{\Delta t_{z}} \tau_{\gamma \gamma} \approx \frac{0.14}{\ln \frac{\epsilon_{u}}{\epsilon_{\mathrm{d}}}} \frac{\epsilon_{u}}{\gamma \Gamma}
$$

As long as the right hand side is $\ll 1$, one can have at the same time $\tau_{\gamma \gamma} \leq 1$ and a high radiative efficiency $\Delta t_{e m} \ll \Delta t_{z}$. In certain BL Lacs, however, as for instance Mkn 421, 
the Klein-Nishina regime is reached and $\epsilon_{u} / \gamma \Gamma \approx 1$; furthermore, if one requires that the jet is not loaded with too many pairs (Ghisellini \& Madau 1996, Sikora et al. 1997), $\tau_{\gamma \gamma}$ has to be $\ll 1$, and a certain degree of fine tuning becomes necessary. In general, however, $\Delta t_{e m}<\Delta t_{z}$. The models which have been fitted to typical $\gamma$-ray blazars have by construction $\Delta t_{z}$ equal to the observed variability time scale, which is several hours; Salvati, Spada \& Pacini (1996), for instance, in the case of $3 \mathrm{C} 279$ find $\Gamma \approx 10$ and $z \approx 10^{17} \mathrm{~cm}$.

\section{GEOMETRY AND RAPID VARIABILITY}

The acceleration of the emitting electrons must occur in situ, and strong shocks are the most likely location. The orientation of the shocks with respect to the jet axis is preferentially at angles $\approx 1 / \Gamma$. This is seen in numerical simulations (Bowman 1994, Gómez et al. 1997), and is due to arcsin $1 / \Gamma$ being the causal angle traced by a perturbation which moves isotropically at speed $c$ in the comoving frame. Also, when the flow is deflected by the external medium, angles around $1 / \Gamma$ are preferred, since at those angles the ram pressure levels off at values close to the internal pressure. Finally, it is plausible that $\Delta t_{i n}$ and $\Delta t_{e m}$ are much smaller than $\Delta t_{z}$.

We can envisage a geometry of the emission region similar to Fig. 1: the accelerating shocks are a series of opening and closing cones; a very thin layer of extra electrons (the perturbation) move down the jet with velocity $\beta c, \beta=\sqrt{1-1 / \Gamma^{2}}$; they radiate immediately after being accelerated, so that the emission region is a ring at the intersection between the electron layer and the conical shocks; if the cone opening is precisely $\arcsin 1 / \Gamma=\operatorname{arcos} \beta$, and the line of sight coincides precisely with one generatrix of the cone, the photons emitted in the observer's direction pile up at the same observed time.

Many of these restrictions can be released. One can assume that the cone opening 
angle $\alpha$ and the viewing angle $\theta$ are different from each other; then the pile up condition becomes

$$
\frac{\beta c}{\cos \alpha}=\frac{c}{\cos (\theta-\alpha)}, \quad \theta=\alpha \pm \operatorname{arcos} \frac{\cos \alpha}{\beta}
$$

Real solutions exist only if $\alpha \geq \operatorname{arcos} \beta$; when $\alpha>\operatorname{arcos} \beta$, the pile up effect takes place along two lines of sight. Also, it is not essential that $\theta$ coincides exactly with the pile up value. Fig. 2 shows the result of viewing angles differing by various amounts from the optimal one. If $x, y$, and $z$ are cartesian coordinates of the emission ring, with $z$ along the jet axis, $s=z \tan \alpha$ is the ring radius, $t$ is the time, and $t_{o b s}$ the time at which a given wavefront reaches the observer, the flux $F$ is given by

$$
\begin{gathered}
F\left(t_{o b s}\right) \propto \int \sqrt{\left(\frac{\partial x}{\partial t_{o b s}}\right)_{t}^{2}+\left(\frac{\partial y}{\partial t_{o b s}}\right)_{t}^{2}} d t \\
x=\frac{c}{\sin \theta}\left[t_{o b s}-t(1-\beta \cos \theta)\right], \quad y=\sqrt{s^{2}-x^{2}}, \quad z=c \beta t
\end{gathered}
$$

The curves have been computed with $\theta=e \times \operatorname{arcos} \beta$ (where $e$ is a number $0<e<1$ ), $\alpha=\operatorname{arcos} \beta, \Gamma=10$, and $\Delta t_{z} \approx 9$ hours. One should note that the vertical axis is logarithmic, and the height of the spikes is very often a factor of several; one should further note that blazars are observationally selected to have $\theta \leq \operatorname{arcos} \beta$, and the a posteriori probability of having $\theta \geq e \times \operatorname{arcos} \beta$ is relatively large, $\mathrm{P}(\theta \geq e \times \operatorname{arcos} \beta \mid \theta \leq \operatorname{arcos} \beta)=1-e^{2}$; for instance, the sharpest peak shown in Fig. 2 should be observable in $19 \%$ of the cases. The peaks appear because the relevant geometry is not the one of the jet as a whole, but instead the one of the emission region, which -in the case of strong radiation lossestraces the surface of the shocks. The preferential orientation of this surface results in the radiation field being strongly anisotropic at any point of the emission region at the time of 
the perturbation; the peak photons are distributed over an angle much narrower than $1 / \Gamma$, of the order of $(1 / \Gamma) \times \sqrt{\Delta t_{e m} / \Delta t_{z}}$; the relevant length is the radiative length, instead of the geometrical one, and one has $\Delta t_{\text {obs }} \approx \Delta t_{e m}=\left(z / c \Gamma^{2}\right)\left(\Delta t_{e m} / \Delta t_{z}\right)$. Both results can be described in terms of an "effective" Lorentz factor $\approx \Gamma \times \sqrt{\Delta t_{z} / \Delta t_{e m}}$, and explain why -at variance with Eq. 1 $-\Delta t_{o b s} \ll \Delta t_{z} \approx \Delta t_{r}$.

Anisotropies in the laboratory frame on scales smaller than $1 / \Gamma$ are equivalent to the loss of isotropy in the comoving frame, at variance with the standard scenario, and may have interesting consequences. While the peak photons, as we have found, are very closely aligned, the emitting electrons interact with the same peak photons at angles of the order of $1 / \Gamma$; this breaks the assumptions under which Eq. 3 was derived, and allows a situation where at the same time the radiative losses are very rapid and the pair production opacity is very low. Also, if the line of sight is not closely aligned with the shock surface, the observer will infer a photon density at the source lower than the one experienced by the electrons; this breaks the assumptions under which synchrotron self Compton models are usually computed, and allows larger ratios between the Compton and synchrotron regions of the spectrum.

We stress finally that the sharp features carry only a small fraction of the total radiated energy; most of this energy resides in the long lived, low level tail, which by construction has a time scale of order $\Delta t_{z}$. So the observed light curve will exhibit conspicuous sharp features only if the injection events are spaced at intervals $\geq \Delta t_{z}$; in the opposite case a given peak will be swamped by the superposition of the many tails of the preceding peaks.

Because of the assumption of negligible radiative lifetime, the curves of Fig. 2 are the Green functions of the variability problem for the given set of parameters, and by convolving them with an arbitrary injection function one obtains the general solution. Fig. 3 is our best fit by this method to the TeV light curve of Mkn 421 on 15 May 1996 with the same 
parameters of Fig. 2 and $e=0.9$. It shows that it is not necessary to assume extreme jets to account for extreme variability; only, similar episodes should not be observed to repeat before times of order $\Delta t_{z} \approx$ several hours. We recall that Mkn 421 was observed to flare with $\mathrm{a} \approx 60$ minute timescale also on 7 May 1996 (Gaidos et al. 1996). With a different injection function we can easily fit this flare as well. Because of the longer duration of the episode, we find that the ratio of the fluxes after and before the flare should have been higher than on 15 May 1996, but there are no data to check this.

\section{CONCLUSIONS}

We have developed a model to explain extremely fast variations in the emission of relativistic jets. Under the following assumptions:

1. the jet has typical parameters $\left(\Gamma \approx 10, z \approx 10^{17} \mathrm{~cm}, \Delta t_{z} \approx 9\right.$ hours $)$;

2. cold electrons are injected in the center and move outwards with bulk velocity $c \beta$;

3. electrons are accelerated in conical shocks with opening angle $\approx 1 / \Gamma$;

4. injection time and cooling time are $\ll \Delta t_{z}$;

5. the viewing angle is $\theta \approx 1 / \Gamma$,

it is possible to observe a very short bump $\ll \Delta t_{z}$, because the component of the peak photons' velocity along the jet is near to the electron velocity, and the photons pile up on the same wavefront. In particular, we have been able to fit with typical jet parameters the 20 minute TeV flare of Mkn 421 on 15 May 1996.

Deviations from the ideal geometry could be some curvature in the shocks, or a non-negligible lifetime of the electrons at low energies; these should result in a certain 
amount of smoothing, which needs to be computed in detail. The extension of this model to the case of non-negligible particle lifetime is currently under investigation with the aim of comparing the expected behaviour of the emission at different frequencies. We may note straight away that in at least one case (PKS 2155-304) the burst has been seen to evolve with frequency-dependent time scales (Urry et al. 1997), roughly compatible with synchrotron cooling in a uniform field. This is possible only if $\Delta t_{r} \ll \Delta t_{z}, r \ll z / \Gamma$ (with far reaching implications on the jet dynamics), or in a non-standard geometry as the one proposed here.

This work was partly supported by the Italian Space Agency through grants ASI-95-RS-120 and ARS-96-66. 


\section{REFERENCES}

Blandford, R.D., Levinson, A. 1995, ApJ, 441, 79

Bowman, M. 1994, MNRAS 269, 137

Gaidos, J.A., et al. 1996, Nature, 383, 319

Dermer, C., Schlickeiser, R., Mastichiadis, A. 1992, A\&A, 256, L27

Ghisellini, G., Madau, P. 1996, MNRAS, 280, 67

Gómez, J.L., Martí, J.M., Marscher, A.P., Ibáñez, J.M., \& Alberdi, A. 1997, ApJ, 482, L33

Hartman, R.C., et al. 1992, ApJ, 385, L1

Inoue, S., Takahara, F. 1996, ApJ, 463, 555

Maraschi, L., Ghisellini, G., Celotti, A. 1992, ApJ, L5

Salvati, M., Spada, M., Pacini, F. 1996, in Gamma-ray Emitting AGN, ed. J.G. Kirk, M. Camenzind, C. von Montigny \& S. Wagner (Heidelbergh: MPI für Kernphysik V37-96), 161

Sikora, M., Madejski, G., Moderski, R., \& Poutanen, J. 1997, ApJ, 484, 108

Sikora, M., Begelman, M.C., Rees, M. 1994, ApJ, 421,153

Urry, C.M., et al. 1997, ApJ, 487, 799 
Fig. 1. - The geometry assumed for the acceleration and emission region. See text for details.

Fig. 2.- The light curves observed for $\alpha=\operatorname{arcos} \beta$ and $e=\theta / \operatorname{arcos} \beta<1$. The values of $e$ are indicated by the labels.

Fig. 3.- A fit to the TeV flare of Markarian 421. The geometrical time scale of the underlying model is $\Delta t_{z}=9$ hours. 


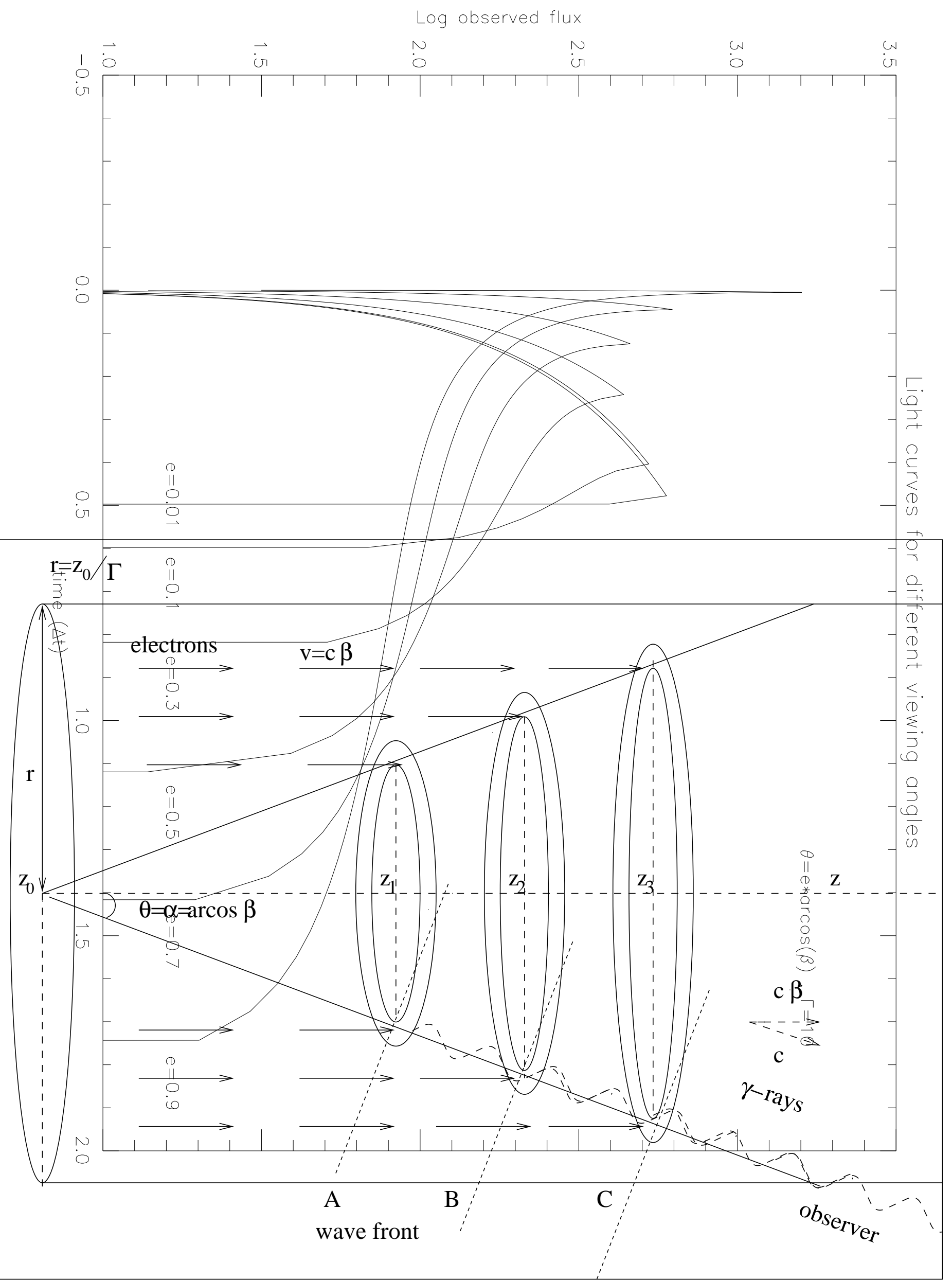




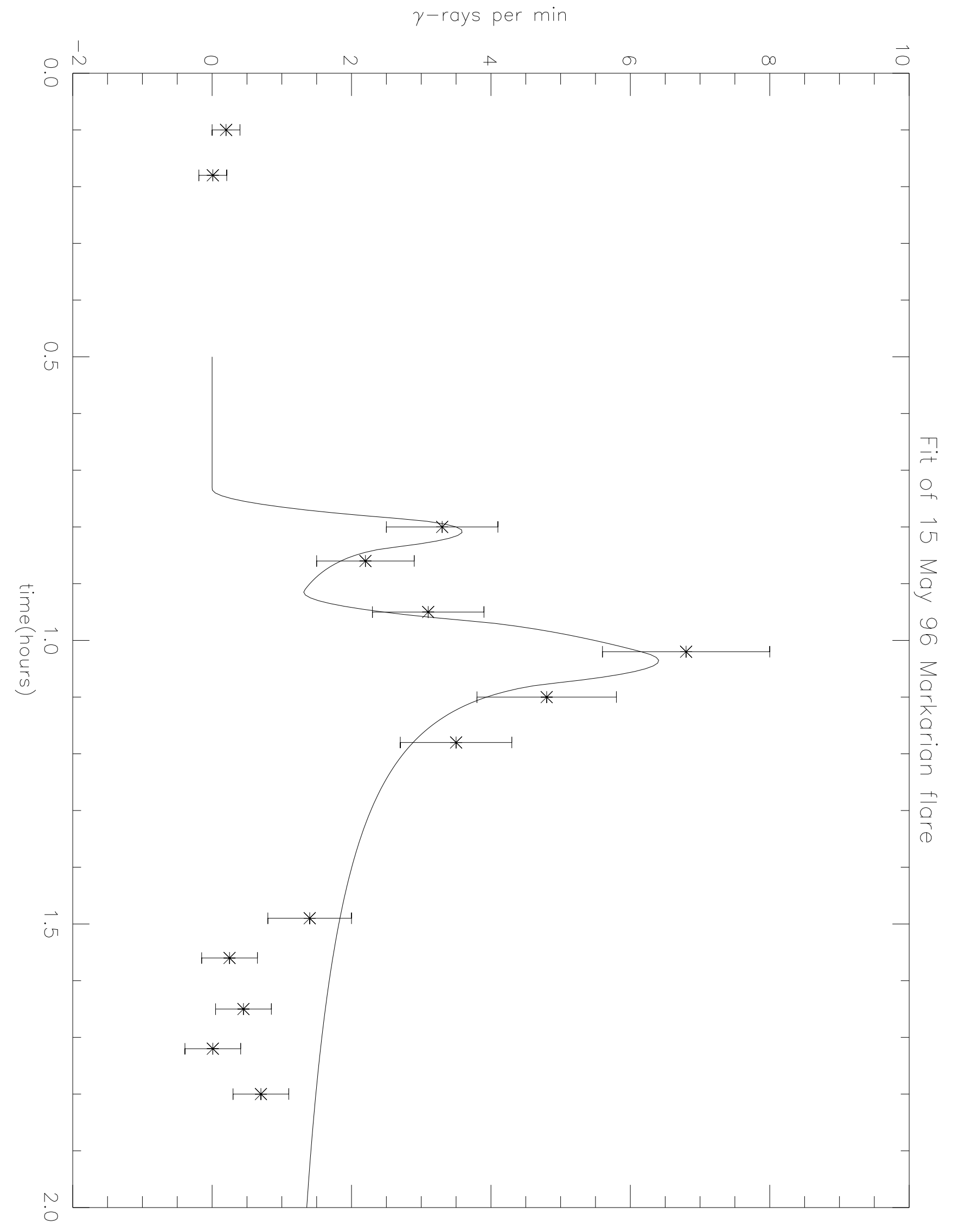

